\title{
Nanobiotechnology in Agricultural Sector: Overview and Novel Applications
}

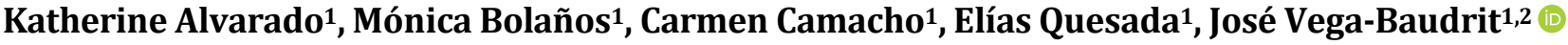 \\ ${ }^{1}$ Chemistry Department, National University of Costa Rica, Heredia, Costa Rica \\ ${ }^{2}$ National Nanotechnology Laboratory LANOTEC-CeNAT-CONARE, San José, Costa Rica \\ Email: jvegab@gmail.com
}

How to cite this paper: Alvarado, K., Bolaños, M., Camacho, C., Quesada, E. and Vega-Baudrit, J. (2019) Nanobiotechnology in Agricultural Sector: Overview and Novel Applications. Journal of Biomaterials and Nanobiotechnology, 10, 120-141. https://doi.org/10.4236/jbnb.2019.102007

Received: November 27, 2018

Accepted: April 23, 2019

Published: April 26, 2019

Copyright $\odot 2019$ by author(s) and Scientific Research Publishing Inc. This work is licensed under the Creative Commons Attribution International License (CC BY 4.0).

http://creativecommons.org/licenses/by/4.0/

\begin{abstract}
The agricultural sector has many problems achieving the desired yield in products because of pathogens, viruses, pesticides, herbicides, temperature, soil moisture, among others. Nanotechnology, along with other sciences, has emerged in industry and society due to the interest in solving problems such as those mentioned above, in order to serve the needs of the world population. The present review discusses the principal topics about Nanobiotechnology, such as generalities, applications, aspects that can be improved and perspectives, beside its influence in the agriculture industry.
\end{abstract}

\section{Keywords}

Component, Nanotechnology, Biotechnology, Agriculture, Nanobiotechnology

\section{Introduction}

Nanotechnology is present in almost all areas of our daily life and is recognized by the European Commission as one of the six "Key Enabling Technologies" that contribute to sustainable competitiveness and growth in many areas of industrial application [1]. There are a variety of concepts about nanotechnology in the literature, however, it can be defined as a novel, innovative and interdisciplinary science field that involves the designing, development and application of materials and devices at molecular level in nanometric scale [2]. In other words, it is a field of applied science and technology which deals/involves in manipulating matter and its atoms and molecules with the purpose of fabricating materials, devices and systems with new characteristics that can improve their applications [3]. The principle of Nanotechnology is to work atom by atom, with the purpose of obtaining structures with new molecular organization and through this, to 
exploit these properties and develop innovative devices [4].

On the other hand, biotechnology uses the techniques of biology in order to manipulate molecular, genetic and cellular processes to develop products and services and it can be used in diverse fields from medicine to food and agriculture [5].

Having said that, the Nanobiotechnology is the integration of nanotechnology, biotechnology and also chemical and physical processing, material science and system engineering with the aim of obtaining new products previously modified at nanoscale that may have improved conditions with respect to the conventional materials [6].

Due to the new chemical, physical and biological properties of nanomaterials and nanoparticles, they have been rapidly exploited in many areas such as agriculture, food, medicine, pharmacy, electronics, material science and energy sectors among others [2] [6].

For many countries, agriculture sector is very important for their economy, including more than $60 \%$ of the population which depends upon agriculture to earn their livelihood directly or indirectly [1].

Agriculture is one of the most important and stable sector because it produces and provides raw materials, mainly for food and feed industries. The limit of natural resources (production land, water, soil), the growth of the population in the world, the increasing global food security and the climate change, claim the agricultural development to be economically further, viable, environmentally and efficiently [2] [5]. For the agriculture, the change in properties is thanks to the possibility to manipulate, change and reduce the atomic and molecular size, what allows to change the interactions between molecules and hence, have materials with new characteristics. The new properties and possibilities of nanotechnology in this area are high reactivity, sensitivity, enhanced bioavailability and bioactivity, adherence effects and different surface effects [7].

Now, after years of green revolution and decline in the agricultural products ratio to world population growth, it is obvious the necessity of employing new technologies in the agriculture industry. Modern technologies such as bio and nanotechnologies can play an important role in increasing the annual production and improving the quality of food produced by farmers. Many believe that modern technologies will secure growing world food needs as well as deliver a huge range of environmental, health and economic advantages [3].

The need of new science and technology interventions have allowed to revolutionize the agricultural industry with novel tools for the molecular management of diseases, rapid disease detection, enhancing the ability of plants to absorb nutrients, improving agricultural productivity and efficiency with lower cost and less waste, among others [4] [8].

To conclude, the objective of this investigation is to elaborate a review about nanotechnology in the agriculture sector, learn about its generalities, important applications and aspects that can be improved. 


\section{History}

The concept of "Nanoscience" has been attributed to Laureate Richard Feynman in 1959 and the term "Nanotechnology" was used for the first time in 1974 by Norio Taniguchi of the Tokyo Science University [7]. After that, the development of nanotechnologies has been attributed to two inventions that have revolutionized imaging and manipulation of nanoscale objects: The Scanning Tunneling Microscope (STM) invented in 1981 and the Atomic Force Microscope (AFM) in 1986. These instruments practically opened the door for scientists to the "nanoworld" and since these discoveries the studies in nanotechnology don't cease to increase in the innovations in electronics and also in the use of nanoparticles in many areas [9].

On the other hand, the studies about nanotechnology in agriculture started growing for industrial applications nearly half a century ago, nevertheless, the impulse to other scientists for its use is attributed to recently reports published, which are focused on identifying the research areas that should be found and also on set the agenda for nanotechnology research in agricultural applications, which became the principal guiding force for many nations that have agriculture as primary occupation of the population [10].

\section{Applications in Agriculture}

Nowadays, the world's population is growing very rapidly, and it is expected to reach 8.5 billion by 2030 [11], which means that the demand for food, natural resources like water, land to establish cities and cultivate, will also increase [12]. In the same matter, the climate change due to the pollution has caused a very negative impact on the crop production [12]. In that sense, the food industry needs to come up with quick and novel alternatives in order to fulfill the heavy demand for food and the shortage of land and water.

Currently, some of the solutions to cope with those problems have been the use of fertilizers, pesticides and genetically modified organisms (GMO) [13]. Nevertheless, the indiscriminate use of chemical compounds to increase the crop production or efficiency, has caused serious issues like heavy soil, air and water contamination, human and animal diseases and destabilization of the ecosystems [12] [14]. For example, related to the overuse of pesticides, many of the pests that affect the crops have developed resistance, analogous to the bacterial antibiotic resistance, which causes that the farmers tend to use more quantity and stronger pesticides, some of them being quite toxic [15].

With that being said, bio and nanotechnology now comes into play, as innovative technologies that may have the solutions to many of the issues that the humans and agricultural industry have been facing [1] [6] [13]. As an illustration, Table 1 shows some of the main materials used in agriculture that have been developed using nanometric technologies [15] [16] [17].

\subsection{Nanofertilizers}

Like mentioned above, fertilizers are chemical substances, like urea, ammonium 
Table 1. Nano materials used nowadays in agriculture [15] [16] [17].

\begin{tabular}{|c|c|}
\hline Material & Purpose \\
\hline Magnetic carbon coated nanoparticles & $\begin{array}{l}\text { Controlled released herbicide and Smart } \\
\text { agrochemical delivery system used in several } \\
\text { plants such as tomato, sunflower and pea }\end{array}$ \\
\hline $\begin{array}{l}\text { Polyhydroxybutyte-co-hydroxyvalerate } \\
\text { microspheres with atrazine } \\
\text { (about } 13 \mathrm{~nm} \text { ) } \\
\text { Inorganic } \mathrm{Zn} \text {-Al layered double } \\
\text { hyroxide (ZAL) as release agent }\end{array}$ & $\begin{array}{c}\text { Nano controlled release system of } \\
\text { 2-4-dichlorophenoxyacetate used as herbicides }\end{array}$ \\
\hline Porous hollow silica nanoparticles & $\begin{array}{l}\text { Nano controlled release system } \\
\text { of wáter soluble pesticide }\end{array}$ \\
\hline $\begin{array}{l}\text { CNT conjugated with INF24 } \\
\text { oligonucleotides } \\
\text { Silver nanoparticles (AgNPs) }\end{array}$ & Reduce rust disease and control pathogens \\
\hline Amphotericin B nanodisks & Treatment of fungal pathogens (proved in vitro) \\
\hline Gold nanoparticles (AuNPs) & $\begin{array}{l}\text { Delivery of DNA during transformation of plant cells } \\
\text { Plant growth promoting rhizobacteria }\end{array}$ \\
\hline
\end{tabular}

salts, phosphates, among others, used to increase the crop production, as they facilitate the access to nutrients required for growing [13] [15]. However, in order to achieve the enormous required amount of food, farmers have resorted to the use of conventional fertilizers at high rates and long periods of time, which is the main anthropogenic factor that has caused the eutrophication of coastal and freshwater ecosystems [18].

In that way, different approaches have been developed to deal with this issue. One of them is the creation of nanofertilizers, that are either nanomaterials that can supply nutrients to the plant, or those that act as enhancers of the traditional fertilizers, but do not directly provide the nutrients [18]. For example, nanoparticles made out of hydroxyapatite, $\mathrm{TiO}_{2}, \mathrm{SiO}_{2}$, or even carbon nanotubes [13].

In regards of the uptake mechanism by the plants, there are two main mechanisms [18]. The first consists on the nanoparticle entering directly into the plant cells through pores on the cell wall, in this case, the size of the nanoparticle needs to be smaller than the pore size (5 to $20 \mathrm{~nm}$ ) [18]. The seconds involves the dissolution of the nanoparticles, which are absorbed through the roots of the plant and release the nutrients as soluble ions [18]. Also, the advantage of using nanoparticles is that the dissolution of these in water or soil is higher compared to the conventional fertilizers, because of its smaller particle size and higher specific surface area [18].

Another strategy has been the development of biofertilizers, in which are used beneficial microorganisms that are able to convert organic matter into compounds that are nutritional for the plant and, at the same time, maintain the soil fertility and its natural habitat [15]. Nevertheless, this approach has a few drawbacks, like short shelf life, lack of suitable carrier materials, high temperature and desiccation sensitivity [15]. Therefore, in order to overcome those issues, 
polymeric nanoparticles for coating to resist desiccation, might be a great application [15]. For example, scientists have investigated the interaction between gold nanoparticles (GNPs) and plant growth promoting rhizobacteria (PGPR) [17]. The results showed a significant increase on growth for some of the strains tested, like P. fluorescens, $P$. elgii and B. subtilis, hence, GNPs have great potential as nano-biofertilizers [17].

\subsection{Nanopesticides}

Some materials contain elements in the nanometer size that claims novel properties associated with these small size, in this way, nanopesticides are defined as any formulation that includes this type of modified materials to generate more efficient products. Since materials vary their properties depending on their size, there is a wide range of nanopesticides that cannot be considered as a single category. For example, some of them are made of organic ingredients like polymers and, in the other hand, some nanopesticides may have inorganic ingredients like metal oxides. It should be noted that the nanomaterials were introduced into pesticides in various forms (particles and micelles); and this nanometric materials are introduced into pesticides to release the active ingredient more slowly and in a selectively way to increase the solubility of the poorly soluble active ingredient $o$ thereby protect the ingredient against premature degradation [19].

In these days, resist environmental conditions such as sun, heat, rain, to be transported through the target and penetrate efficiently the organism (fungus, insect, etc) or to resist defense of the pest/pathogen, are some of the more important characteristics of an ideal pesticide. Even so, pesticides do not have such good chemical characteristics, that is why, now nanomaterials are implemented in this products. Likewise, it has been observed that in economic terms, introducing nanoparticules into pesticides provide monetary, social and environmental benefits by formulating more controlled modes of action. To achieve this, nanomaterials have potential for developing safer chemical pesticide formulations for applications in the field.

Another point to take into account is that nanotechnology has been incorporated into pesticides through the use of nanosuspensions, nanoparticles and polymeric nanoparticles. Nowadays, these forms of introduction into the nanopesticides have been tested to observe their efficiency and effectiveness [15].

Among the main nanomaterials used to improve the properties of pesticides are found the iron oxide nanoparticles and gold nanoparticles, they are mostly used because their easily way to be synthesized. According to the size, the pharmacokinetic parameters can be altered shape and functionalization of the surface of the nanomaterials [20]. That is why, nanotechnology can take an important part in the development of sustainable agriculture, since this technology can control the delivery of nutrients and monitoring the water quality used in this field [5]. As a consequence, it is important to encourage the use and spread of nanomaterials in this aspect. 
Nanopores open up opportunities for agriculture by the improvement of new productivity using research in nanotechnology. For example, Zeolites are nanocapsules of water, herbicides or fertilizers used for efficient dosing and slow release of the vector for pest management. The manipulation of nanoparticles makes possible the arrangement of atoms, modifying shapes, sizes and functions. This allows the manufacture of advanced objects such as Zeolites for the controlled release of active agents such as pesticides [21].

Reducing waste and labor costs are some of the benefits that nanotechnology promises by streamlining agricultural management. Therefore, nanopesticides increased average crop yield by $20 \%$, sunflower by $50 \%$; rice by $35 \%$ and $\mathrm{cu}-$ cumber by $25 \%$. Nowadays, some researches shows that nano-iron oxide pesticides increased rice yields by $25 \%$ and soybean yields increased $48 \%$ [22].

\subsubsection{Management of Insect Pest}

Nanoscale science and engineering have an important role in the creation of agricultural system for the generation of safer food under more controlled production. Although commercial advances and technological impacts are limited due to the relative novelty of the use of nanotechnology in agriculture and food systems, some encouraging results have been obtained. From the perspective of food safety, nanotechnology has a lot to offer, such as the control of insect pests [23].

Insects are one of the most known animal species around the world. They are found in all types of environment. Insects fed on all kinds of plants including crop plants, forest trees, medicinal plants and weeds. They also infest the food and other stored products in warehouses, bins, storage structures and packages causing huge amount of loss to the stored food and also deterioration of food quality [6].

The effectiveness of metal nanoparticles against plants pathogens, insects and pests were proven in previous studies. Hence, nanoparticles can be used in the preparation of new formulations like pesticides, insecticides and insect repellants [6] [15].

\subsubsection{Antifungal Properties of Nanomaterials}

The efficiency of nanoparticles on the control of the growth of some bacteria and fungi on plants has been proven. New Silver nanoparticles (SNPs) have been studied as pesticides for fungal and bacterial control; this is because by having such a small size they are able to penetrate the wall and cell membrane in large scale in biological processes. The antibacterial activity of silver ions has been well established. These SNPs have fungistatic, bacteriostatic and plasmonic properties that are eco-friendly inhibitors against plant-pathogens compared with synthetic fungicides [24]. SNPs can attack membranes and structures denaturing cell. This nanomaterial disrupts transport systems, such as ion efflux, causing a rapid accumulation of silver ions, interrupting cellular processes such as respiration and metabolism by reacting with the molecules [24] [25]. 
In the same way, in terms of nanotechnology, studies have been published on copper nanoparticles and copper oxide nanoparticles for the phytopathological and phytosanitary application [26]. Another nanoparticle used to control fungal pathogens is the chitosan, a natural compound that has several applications in biology, because of its capacity of being biodegradable, safe and non-toxic, environmentally friendly and low-cost production. Some investigations found that chitosan has antifungal properties because the interaction between amino groups of chitosan and negative charges of the macromolecules at the outside of the fungal cell wall [27].

\subsection{Nanosensors}

Agricultural production continues to be constrained by biotic and abiotic factors. For instance, insect pests, diseases and weeds cause considerable damage to agricultural production [6]. Regarding this, Nanotechnology has allowed the use of nanosensors, which are sophisticated instruments that can respond to physical, chemical or biological aspects and transfer that response into a signal that can be used by humans, allowing to detect many factors due to drought, temperature, insect or pathogen pressure, or lack of nutrients [5]. Many years ago the people used traditional techniques such as gas or liquid chromatography and mass spectroscopy instead of nanosensors, however, these devices have the advantages of being small, portable, sensitive with real-time monitoring, precise, quantitative, reliable, accurate, reproducible, robust and stable. Because of that, an alternative to analytical methods could not only improve the efficiency in the investigations but also reduce the cost associated with the traditional methods; the unique and different characteristics of nanomaterials, such as size/shape-dependent optical property, easy and fast turning in surface properties and catalytic functions are very useful for the signal generation and signal amplification [28] [29]. Moreover, the possibility of on-site monitoring allow farmers to closely monitor environmental conditions for plant growth and crop protection and also can contribute to increase the productivity and decreased the use of agrochemicals such as antibiotics, pesticides and nutrients by early intervention; besides that, people can utilize inputs more efficiently by indicating the nutrient or water status of crop plants over fine spatial and temporal scales and through this, apply nutrients, water, or crop protection (insecticide, fungicide or herbicide) only where and when necessary [5] [15].

These smart sensors in the agriculture sector are considered a powerful tool for track detect and control animal and plant pathogen, mainly because the devices help in the detection of chemical contaminants, virus and bacteria in agricultural systems, nutrient content and plant stress [20].

Some specific applications of these sensors in agriculture are the MEMS (Micro Electro Mechanical Systems) that through microelectronic circuits are capable of sensing and monitoring temperature and moisture in soil, to diagnose oil disease (caused by infecting soil microorganisms, such as viruses, bacteria and 
fungi) via the quantitative measurement of differential oxygen consumption in the respiration of good microbes and bad microbes in the soil. Also biosensors are developed, using photosystem II, known to bind several groups of herbicides and chemicals, among others [7].

Through the development of new devices, farmers and population will be benefited in aspects such as economic gain, reduced environmental pollution and lower labor costs [30].

\subsection{Nanobiosensors}

Physical and chemical new properties of nanoscale materials are also exploitable in the development of biosensors. Nanomaterials are used in biosensors with the purpose of improve their sensitivity and efficiency through new signal transduction technologies [5]. Nanosensors with immobilized bioreceptor which is selective for target analyte molecules are called nanobiosensors. In other words, this is a modified version of a biosensor that incorporates a biological derived sensitized element linked to a physical-chemical transducer. This device integrates a biological element with an electronic component to yield a measurable signal and in the other hand, the biological recognition is through the transducer process [5] [31].

The conventional nanobiosensors have three principal components (Figure 1). The probe or biologically sensitized elements such as enzymes, antibodies,

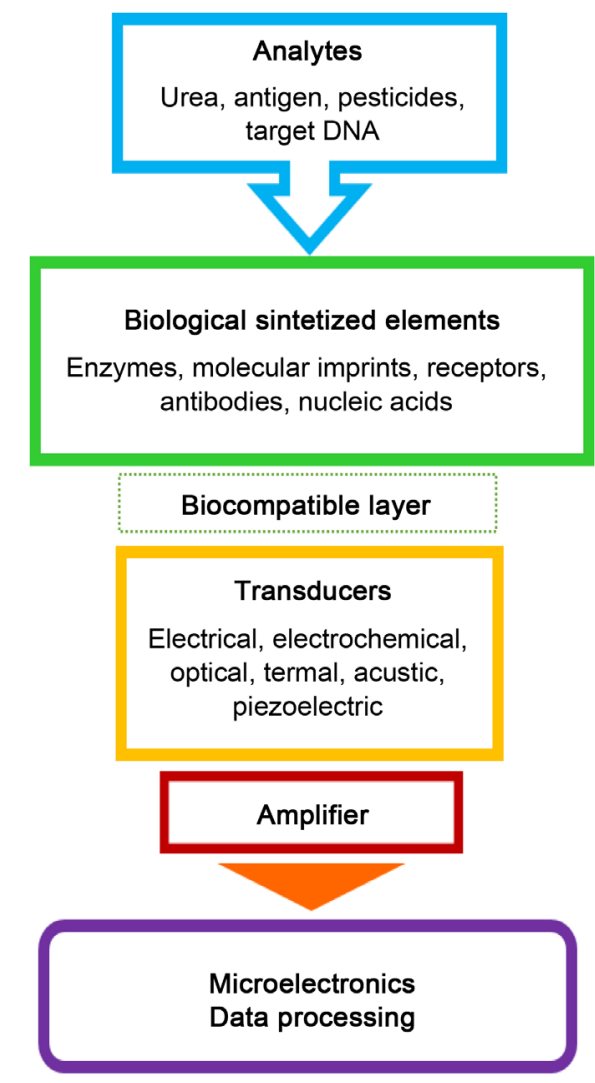

Figure 1. Principle of a nanobiosensor [31]. 
tissues, microorganisms (pathogens), nucleic acids, among others, which have the function of receive signals from the sample and transmit it to a transducer.

The transducer measures physical changes occurring at the biological receptor. After that, the device transforms that energy into measurable electrical emission. The detector, catches the signal from the transducer and then it is passed to a microprocessor where are amplified and analyzed. Finally, the data is transferred to a display and the user can observe the results [31].

Due to investigations that have been carried out in the last years, nanobiosensors can be classified into many types such as mechanical, optical, electronic, viral, probes encapsulated by biologically localized embedding (PEBBLE), nanoshell and enzyme-based nanobiosensors (Table 2). The use of each type of nanobiosensor depends on the analyte, for example, biosensors are used for the detection of phenols, polyaromatic compounds, halogenated pesticides and volatile organic compounds (VOCs) while electrochemical sensors can be used as chemical gas sensors [32].

Their applications include detection of analyte like urea, glucose, pesticides, also the monitoring of metabolites and detection of various microorganisms/pathogens [7]. Regarding the latter, one example is a novel microbial detection technology based on silica nanoparticles has been developed in the last years. The NPs $(60 \mathrm{~nm})$ are filled with a fluorescent dye and conjugated to an antibody specific to a surface antigen of the microbe of interest [15]; this method

Table 2. Types of nanobiosensors used in agricultural sector [30] [31] [32] [33].

\begin{tabular}{|c|c|c|}
\hline Type of nanobiosensor & Samples & Advantages \\
\hline Mechanical & $\begin{array}{c}\text { Nanocantilever, acoustic } \\
\text { biosensors }\end{array}$ & $\begin{array}{l}\text { Highly mass sensitive, more the size } \\
\text { decreases, more the mass reduces }\end{array}$ \\
\hline Optical & $\begin{array}{c}\text { Surface plasmon resonance } \\
\text { (SPR), LSPR, FRET, SERS, } \\
\text { evanescent field based } \\
\text { devices, photonic } \\
\text { crystal devices }\end{array}$ & $\begin{array}{l}\text { Extremely sensitive, small } \\
\text { volumes required, } \\
\text { remarkable optical properties }\end{array}$ \\
\hline Nanowire & $\begin{array}{l}\text { Boron-doped silicon } \\
\text { nanowires (SiNWs) }\end{array}$ & $\begin{array}{l}\text { Analyte independent, sensitive, } \\
\text { real time and quantitative fashion, } \\
\text { biological and chemical } \\
\text { species can be used. }\end{array}$ \\
\hline Electronic & Lab on a chip & $\begin{array}{l}\text { Each chip contains multiple sensors, } \\
\text { hence different target DNA } \\
\text { molecules can be addressed }\end{array}$ \\
\hline PEBBLE & $\begin{array}{c}\text { Poly } \\
\text { (decyl methacrylate)-based } \\
\text { fluorescent PEBBLE }\end{array}$ & $\begin{array}{l}\text { Monitoring inter and intracellular } \\
\text { ions and molecules in real time, } \\
\text { high reversibility and stability to } \\
\text { leaching and photo bleaching. }\end{array}$ \\
\hline Nanoshell & Gold nanoshells & $\begin{array}{l}\text { Rapid assays, enhance } \\
\text { chemical sensing }\end{array}$ \\
\hline Enzyme-based & $\begin{array}{l}\text { 4-Hydroxyphenylpyruvate } \\
\text { Dioxygenase Enzyme }\end{array}$ & Precise and low cost techniques \\
\hline
\end{tabular}


has potential in agriculture sector for the detection of plant pathogens.

Nanobiosensors have been used because of some advantages they have with respect to traditional sensors, such as ultra-sensitivity, what allows the easy detection of virus, small particles and low concentrations of substances that could be potentially harmful; also these devices work at atomic scale with highest efficiency and have increased surface to volume ratio. Nevertheless, it should be mentioned that because of their sensitivity, nanosensors are error prone and are still under early stage [31] [34].

\subsection{Disinfectants}

Elimination of contaminants (such as bacteria, fungi, and viruses) is the first step of any successful investigation on in vitro plant tissue culture. One of the major problems of in vitro plant culture techniques is chronic contamination by microorganisms [35]. Frequent application of conventional antibiotics and chemotherapeutic agents may be phytotoxic or may retard plant tissue growth [35].

In this regard, using AgNPs (with the size range of $35 \mathrm{~nm}$ ) solution after surface sterilization has showed acceptable influence on the control of bacterial infection without any adverse effects on the growth characteristics of regenerated valerian plantlets. However, there are some reports on ineffectiveness of AgNPs on fungal contamination, likely due to AgNPs size. Antifungal activity of AgNPs is size dependent, the smaller is the better [35].

On the other hand, the use of nanoparticles has been proposed as an alternative for plant disinfection. The mechanism of action consists of negative electrons resulting from the excitation of nanoparticles. This can also be used as a disinfectant of bacteria, as when bacteria make contact with nanoparticles, the excited electrons are injected into their bodies, which results in the bacterial removal from the object concerned, as in fruit packaging and food engineering [36].

\subsection{Genetic Material Delivery}

Genetic transformation is a major branch of plant biotechnology [35]. Delivery of genetic material such as DNA and small interfering RNA is important for the development of pest, pathogen and stress resistant strains of crop plants by alteration of gene expression [15]. Gene delivery systems for plant transformation face obstacles such as targeting of delivery system, transportation through the cell membrane, uptake and degradation in end lysosomes and intracellular trafficking of DNA to the nucleus [15].

Nanobiotechnology offers a new set of tools to manipulate the genes using nanoparticles, nanofibers and nanocapsules. Properly functionalized nanomaterials serve as transportation and could carry a larger number of genes as well as compounds able to trigger gene expression or to control the release of genetic material throughout time in plants [7].

Particle bombardment and Agrobacterium mediated transformation are two 
applicable procedures for genetic transformation in plants albeit, it was shown that Agrobacterium mediated transformation was notably efficient with the lowest level of gene copy number. Moreover; Agrobacterium mediated transformation derived plants show a more stable transgene expression and low transgene silencing. Nevertheless, in this technique, removal of surplus bacteria after cultivation with conventional antibiotic is challengeable [35].

On the other hand, gene gun or particle bombardment is one of the popular tools to deliver DNA into intact plant cells. Particles used for bombardment are typically made of gold since they readily adsorb DNA and are non-toxic to cells [37]. Experiments showed that the plasmid DNA transferred by gene gun method using gold capped nanoparticles was successfully expressed in intact tobacco and maize tissues [7].

However, gold NPs (5 - $25 \mathrm{~nm}$ ) embedded carbon matrices were employed for the delivery of DNA during transformation of plant cells that carried higher amount of genetic material as compared to the microparticles. NPs had lower plasmid and gold requirement as compared to the commercial micrometer-sized gold particles. Moreover, plant-cell damage with the NPs was minimal with increased plant regeneration [15].

The major advantage is the simultaneous delivery of both DNA and effect or molecules to the specific sites that results in site targeted delivery and expression of chemicals and genes respectively [7].

Controlled biochemical manipulations in cells have been achieved through the integration of carbon nanofibers which are surface modified with plasmid DNA [7].

Carbon nanotubes (CNTs) could also be regarded as a potential gene delivery vector to plant cells. Single-stranded DNA wrapped around single wrap carbon nanotubes was able to access the cytoplasm of walled plant cells. This could be a promising observation to introduce short dsRNA pieces into the plant cell nucleus for small RNA-induced gene activation. Protoplasts could also be a target for gene delivery where larger CNTs with adsorbed plasmids would be employed to introduce genes-of-interest into the plant genome [38].

In addition to these vectors, nano-fibers/nanoparticles/nanovesicles with cationic nature have ability to precipitate negative charged DNA molecules and bring into cell through nonporous of cell membrane. This process has similarity with microinjection method of gene delivery [39]. Nanoparticulate cationic liposomes and cationic polymers are pursued as alternatives. Due to their permanent cationic charges interact electrostatically with negatively charged DNA to form complexes (lipo- or polyplexes) [15].

Although liposomes formed from cationic phospholipids offer several advantages as vectors for gene delivery into plant cells such as ease of preparation, enhanced delivery of encapsulated DNA by membrane fusion, protection of nucleic acids from nuclease activity, targeting to specific cells, delivery into a variety of cell types besides protoplasts by entry through plasmodesmata [3] [15]. Their 
toxicity and relative low transfection efficiencies are disadvantageous as gene delivery vectors [15].

Polymer/DNA complexes are more stable than those involving cationic lipids, and they protect DNA against nuclease degradation. Cationic polymers such as chitosan are used to condense and deliver DNA both in vitro and in vivo. Biocompatibility, low immunogenicity and minimal cytotoxicity render chitosan NPs $(100-250 \mathrm{~nm})$ a good alternative to viral or lipid mediated transfection [15].

The ability of surface functionalized mesoporous silica nanoparticles (MSNs) to penetrate plant cell walls also opens up new ways to precisely manipulate gene expression at single cell level by delivering DNA and its activators in a controlled fashion [37].

Plasmid DNA molecules carrying a reporter gene can be encapsulated in micrometer-sized calcium alginate beads referred to as "bio-beads". These bio-beads have a possibility for efficient transformation in plants [3].

The major advantage of this technology is the simultaneous delivery of both DNA and effectors molecules to the specific sites that results in site targeted delivery and expression of chemicals and genes respectively. This is how the nanoparticle mediated plant transformation differs from the conventional genetic engineering methods (like electroporation, microinjection, etc.) [37].

\subsection{Bar Coding}

Barcodes are referred as a small symbol which is used for fast and easy identification. It contains a portable device which is powered by batteries and interfaced with laptop or computers. It is multiplexed with various pathogen detectors and has the potential to detect different pathogens simultaneously [39].

The applications of nanobarcodes include: DNA nano barcoding for pathogen detection (such as Ebola and SARS); monitoring of bacterial systems; can be used as a "nano-sensor" to detect water levels, soil nutrient information, and chemical levels; tagging food packages (Pathogens, Temperature changes, Leaks); biomolecular tracing; maximizes productivity; monitoring the quality of agricultural products [39] [40].

Moreover, DNA barcoding methods are a simple, rapid, and low-cost method for simultaneously detecting the presence of multiple bacteria and pathogen targets in the environment and foods [41]. Novel genes are being incorporated into seeds and sold in the market which can be traced with the help of nanobarcodes that are encodable, machine-readable, durable and sub-microsized taggants. Disease spread through seeds and many times stored seeds are killed by pathogens [7].

South Korean researchers have developed a nano-DNA barcode system, which have a specific nano particles adhere products. The small triangle of color through nanotechnology, not only be visible signs of physical security, but also be embedded in the barcode with nano materials ink. And the newly developed 
nanoparticles invisible barcode, effectively preventing the barcode from fraud, may give us real origin information by food traceability system [42].

\subsection{Nanolaminates}

The largest postharvest losses in fruit and vegetable crops are due to deterioration caused by microorganisms after harvest and during cold storage. Fruits, due to their low $\mathrm{pH}$, higher moisture content and nutrient composition are very susceptible to the attack of fungi, while vegetables are generally less acidic, and their spoilage is usually by bacteria. Although it is very difficult to determine the full extent of postharvest losses due to decay (i.e., attack by microorganisms and physical damages), it is well known that these losses are significant [43].

Therefore, food packaging is one of the main focus of polymer nanotechnology and thus one of the major applications of nano-coatings once it is believed that this technology can implement and improve the principal functions of existing packaging. Depending on the type of food, packaging materials need to satisfy different requirements in terms of light, moisture, water vapor and gas (i.e. oxygen and carbon dioxide) barriers in order to avoid color or taste deterioration, loss of nutritional value and eventually growth of potential pathogens [44].

Nanolamination is a technique for protecting the food from moisture, lipids and gases. Moreover, they can improve the texture and preserve color and odor of the food [45]. Therefore, the application of nano-coatings can be of great usefulness for food packaging once their significantly reduced thickness may improve barrier properties while exchanging their resistance, flexibility and tension, as well as the food appearance. Moreover, nano-coating materials may provide other active functionalities such as antimicrobial activity [44]. The resulting properties of nano-laminate coatings such as mechanical properties, gas permeability and swelling and wetting characteristics are influenced by the kind of adsorbing materials utilized and also by the sequence, the total number of layers and the conditions used for preparation (e.g., temperature, $\mathrm{pH}$ and ionic strength) [43].

Nanolaminates are made up of two or more layers of nano-sized $(1-100 \mathrm{~nm})$ thin foodgrade films which are used on a wide variety of foods: fruits, vegetables, meats, etc. These are prepared from edible polysaccharides, proteins, and lipids [45]. Studies show that polysaccharide- and protein-based nanolaminates are good barriers against oxygen and carbon dioxide, but poor in protecting against moisture. Whereas, lipid-based nanolaminates are good at protecting food from moisture [4].

Other materials used for the nano-coatings, applied in a continuous process as a thin amorphous film of $50 \mathrm{~nm}$ or less, include titanium dioxide. Another example is that of nano-selenium, which is being marketed as an additive to a tea product in China for a number of (proclaimed) health benefits [46].

Previous studies have described the design and potential for use of emulsion-based nano-laminated biopolymer coatings produced using electrostatic 
deposition to produce novel encapsulation and delivery systems to control the bioavailability of bioactive lipids. They also described the use of in vitro and in vivo experiments necessary to study the efficacy of such coating systems. Findings included the utility of a chitosan coating to physically or chemically protect an encapsulated bioactive component within a food product and achieve release and bioaccessibility in the digestive tract after ingestion [47].

Current developments include safety and quality indicators that can be applied as labels or coatings to add an intelligent function to food packaging. For example, to monitor the integrity of the packages sealed under vacuum or inert atmosphere by detecting leaks, freeze-thaw-refreeze scenarios by detecting alterations in time-temperature, or microbial safety by detecting the deterioration of foodstuffs. This area of application is likely to see a rapid growth in the future [46].

In this matter, whey protein nano-coatings can also act as sensors mainly intended to monitor the condition of packaged food or the environment surrounding the food. This technology can provide real-time status of food freshness by using a visible indicator (i.e. thousands of nanocoatings may be designed to fluoresce in different colors upon contact with food pathogens, chemical contaminants, or toxins in response to changes in environmental conditions e.g. $\mathrm{pH}$, temperature and moisture) [44].

It is suggested that barrier properties of nano-laminate coatings are improved due to their nano-structure, which has an increased tortuosity resulting from the electrostatic interactions between the nano-laminate's components and also from the interpenetration of the successively deposited layers that hampers gas molecules migration through the structure [43].

Furthermore, nanoparticles can have a dual purpose: besides acting as carriers of additives, they may also provide improvements in the mechanical and barrier properties of the structures where they can be incorporated. However, the efficiency of nanolayer systems with a variety of features (e.g., antioxidant, antimicrobial and reduction in gas exchange) still remains little studied [43].

\subsection{Nanoremediation}

As previously mentioned, pesticides are an essential key part of agriculture, since they allow the growth of crops by protecting them from pests that causes major loses on yields [48]. Unfortunately, most of the pesticides being used nowadays doesn't completely reach the target and the leftovers end up contaminating the surrounding soil, water and air resources and, finally, affect other living beings like animals, plants and humans [49] [50]. For example, arsenic, chlorpyrifos and benfuracarb are some of those pesticides being used that represent a huge health risk on humans, since they are neurotoxic, cytotoxic and carcinogenic [48] [49] [50].

With that being said, one major challenge that humans face these days is the restoration of contaminated soil, water and air to reach a quality level in which 
they no longer represent an environmental and health harm [51] [52]. Nevertheless, traditional remediation techniques are not so effective, quite costly, laborious and may also produce its own contamination [50] [51] [52]. As a promising alternative, nanoremediation has been investigated and might be a less costly and more effective solution to conventional remediation, as it relies on certain nanomaterials, like nanoparticles, which have the capacity to react with the pollutant, enable the transformation into less toxic compounds or immobilize it by adsorption [52].

Also, nanoremediation, compared to traditional remediation, offers some advantages, like less time to complete the cleanup process, since the NPs used have higher surface area and, hence, are more reactive [50] [51] [52] [53]. Also, the use of nanomaterials provides more technologies for in situ remediation, which is less expensive, and avoids the necessity to excavate into the ground, the disposal of contaminated soil or "pump and treat" for groundwater [28] [50].

For instance, nanoscale titanium (IV) oxide is being investigated for the photocatalytic degradation of contaminants in air and water, like VOCs or NOx [52]. Also, zero valent iron nanoparticles, also known as nZVI, have been widely investigated in order to develop novel nanoremediation techniques [28] [49] [50] [51] [52] [53]. These NPs are good electron donors and have shown capacity to remediate or degrade pesticides such as atrazine, lindane, chloroacetanilide, molinate, malathion, chlorpyrifos, dinoseb, organochlorine pesticides and heavy metals like arsenic, lead, mercury and zinc [49] [52]. Nevertheless, there are also concerns because scientists haven't fully investigated the behavior, toxicity and impact on biological systems of the nZVI once they're applied [28] [52].

\section{Impact in Agriculture: Pros and Cons}

As described above, nano biotechnology is characterized for designing and developing innovation materials at nanometer level for application in diverse areas. Following this aspects, Nanotechnology presents great advantages for the development of new technologies according to agricultural requirements (Figure 2) [1].

The possibilities of innovation have increased after the incorporation of emerging technologies such as nano and biotechnology, which impulse the search of new market opportunities after the discovery of tools that improve areas of global and commercial importance such as agriculture [54].

The use of this technology implies, in turn, the efficient use of resources and time in the generation of research within agriculture, such as reproductive science and technology, conversion of agricultural and food waste to generate energy and other valuable byproducts, disease prevention and treatment of common diseases and reducing the negative impacts of pollution due to the excess use of pesticides and fertilizers [32].

Through technology, nanoscience has shown great potential in improving food safety, quality, product traceability, nutrient delivery enhancing, packaging performance, and improving agricultural and food processing [36]. These aspects are areas of application and development of agriculture in current times, 


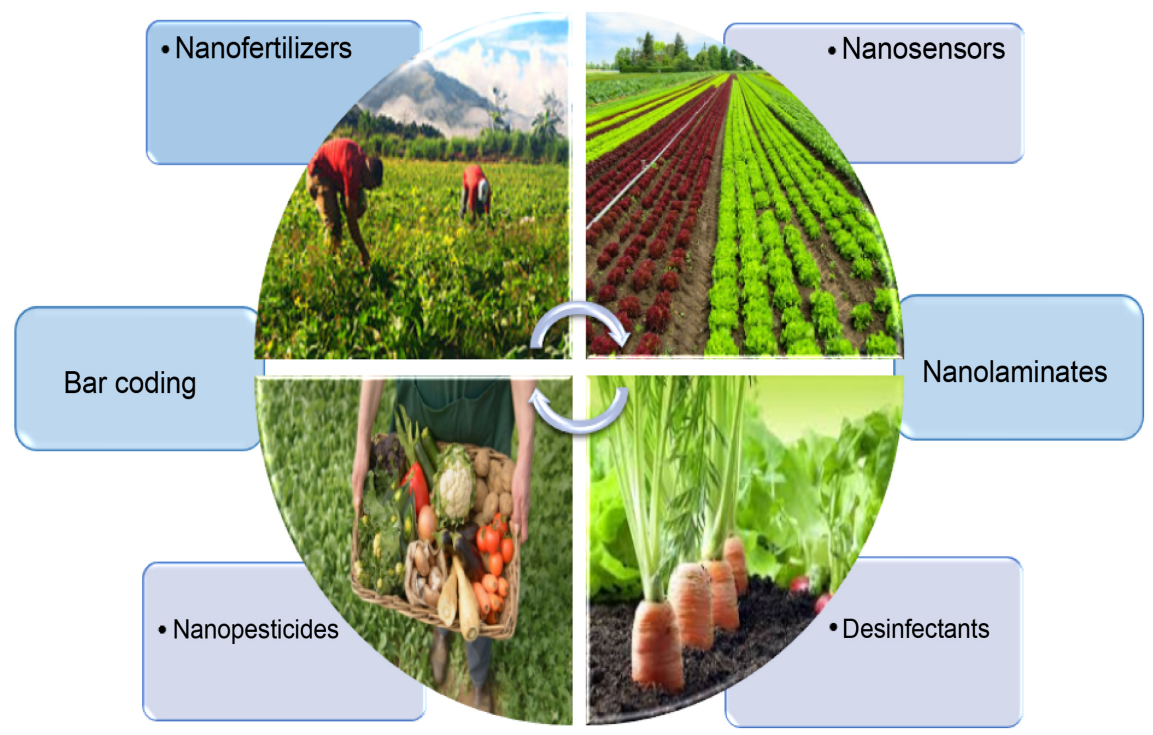

Figure 2. Some applications of Nanobiotechnology in the agricultural sector.

nanotechnology present great advances in the use of resources, reduction of environmental impact and search for environmentally friendly technologies.

Modern agriculture has been shaped by the use of technology within basic agriculture. Among the latest line of technological innovations, nanotechnology is occupying an important place in the transformation of agriculture. The development of nanodevices and nanomaterials could open new applications in plant biotechnology [5].

As detailed above, applications of nanomaterials in this areas includes the germination and faster production of the plant, effective protection of plants with a reduced environmental impact, as opposed to traditional approaches. In addition, intelligent nanosensors are a viable option to detect pesticide residues in the field [28].

In the other hand, studies have shown that, using nanomaterials is not intrinsically risky; but the use of certain nanometric materials in agriculture, water and food have risks for human use and consumption, for the environment or for both since their long-term impact has not been proven, such as the bioaccumulation of these materials. Likewise, is important to consider that biomagnification and biotransformation of nanoparticles genetically modified in food crops are not yet well known. In addition, a good regulation of the entry of these materials into the food chain must be taken into account, or else, the manufactured nanomaterial may represent a potential risk to humans, sand and animals [7].

The use of nanotechnology in agriculture offers great potential for significantly higher productivity and agricultural efficiency at a lower cost, less use of pesticides and less waste of resources. Even so, is important to highlight that the appearance of these applications in agriculture and other sectors has also raised concerns about the impact of the use of these technologies on the safety of environmental and human health. Nanotoxicology, defined as the study of the toxi- 
cology of the main components of nanometric materials, has developed an effort to answer critical questions about the hazard, the exposure and the ultimate risk [8] [55].

In the same way, in the use of nanoparticles, diffusion in nature and a possible toxicity must be controlled, size impact of the use of these components within the area of agriculture for the innovative development of technologies in this area.

\section{Future Perspectives}

Currently, the main objective of nanotechnology research focuses on applications in the field of electronics, energy, medicine and life sciences, areas that are commonly in constant investigation for their impact at the social and commercial level. On the other hand, agriculture is not considered a powerful industry, so the research directed to these issues is considerably smaller. While nanochemical pesticides are already in use, other applications are still in their early stages, and it may be many years before they are commercialized or reach the public [5]. Due to this there is a broad path for the study of new research areas within agriculture.

Since nanobiotechnology is use to develop advanced tools in agriculture, the toxicity of nanoparticles is a recurrent theme in many research studies. Integrating "sustainable development" and considering the "environmental impact" are the challenges that nanotechnology has for the use of new resources within agriculture [9].

Studies have shown that the majority of companies dedicated to capital for research in nanotechnology focus on the search of devices with high technological demand and therefore monetary. Consequently, concerns in agriculture are still not a priority, so studies should be conducted on the importance and economic impact of nanotechnology within this area.

So far, ethical and social issues about nanobiotechnology have not been an important issue for many researchers in this area. Therefore, it is proposed to generate initiatives to anticipate the ethical and social problems that may arise during the development of nanobiotechnology, in order to respond proactively and responsibly to the probable public, media and political problems [56].

Likewise, highly detailed information of how plants respond to exposure and the molecular-level effects when nanomaterials are introducing into the plant and the correlations between physiological and biochemical parameters should be provided to bring more complete understanding effects of toxicity. Between the information that must be provided includes aspects such as the study of low dose exposures with sensitive endpoints, transgenerational studies, impacts on nutritional quality, co-contaminant effects and the impacts of exudation and microbial activity on particle fate and dynamics [7]. As shown in Figure 3, nanoparticles must have specific characteristics to be used in agriculture so they must be guaranteed for their consequent use within this industry [57]. 


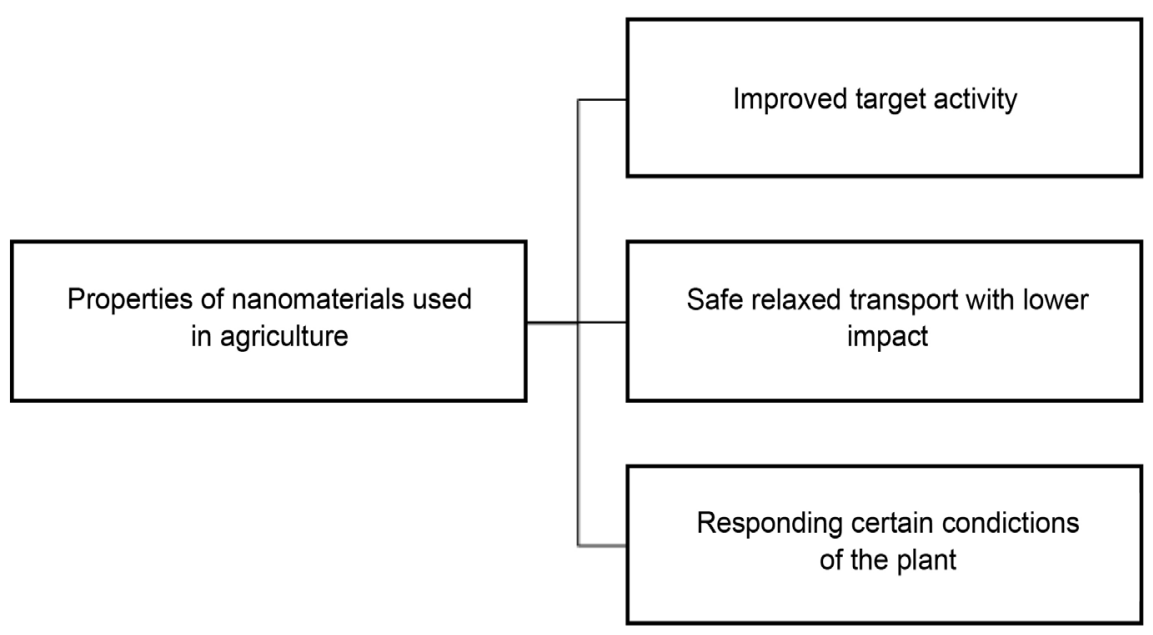

Figure 3. Properties that nanomaterials must have for their use in agriculture [57].

\section{Conclusions}

Nanobiotechnology is characterized as a science with innovative features that has the possibility to generate high technology tools for the development of sustainable fields such as agriculture. Among these features are the development of materials in nanometric scale $(1-100) \mathrm{nm}$ that have versatile characteristics according to their size and manufacturing form such as hydrophobicity, permeability, $\mathrm{pH}$, etc.

For the agriculture sector, the properties and possibilities of nanotechnology in this area are high reactivity, enhanced bioavailability and bioactivity, adherence effects and surface effects of nanoparticles. Nanotechnology comes as an innovative technology that may have the solutions to many of the issues that the humans and agricultural industry have been facing.

Among the predominant areas in which nanotechnology has developed in agriculture in recent years are nanofertilizers, nanopesticides, disinfectants, genetic material delivery, nanolaminates, nanosensors, irrigation, bar coding, etc. Using this technology, modern agriculture has been shaped and nanotechnology is occupying an important place in the transformation of agriculture. The development of nanodevices and nanomaterials could open new applications in plant biotechnology.

Even so, it is important to highlight that the appearance of these applications in agriculture and other sectors has also raised concerns about the impact of the use of these technologies on the safety of environmental and human health.

Therefore, it is important to develop adequate studies about the possible toxicity of nanomaterials on agriculture so that its proper use is made possible in the improvement of the processes that this field requires in the future. Likewise, it should be made aware of researchers and investors about the importance of developing technological tools within agriculture to reduce the use of natural resources and the environmental impact caused by the excessive use of chemicals. 


\section{Conflicts of Interest}

The authors declare no conflicts of interest regarding the publication of this paper.

\section{References}

[1] Ali, M.A., Rehman, I., Iqbal, A., Din, S., Rao, A.Q., Latif, A., et al. (2014) Nanotechnology, a New Frontier in Agriculture. Advancements in Life Sciences, 1, 129-138.

[2] Parisi, C., Vigani, M. and Rodríguez-Cerezo, E. (2015) Agricultural Nanotechnologies: What Are the Current Possibilities? Nano Today, 10, 124-127. https://doi.org/10.1016/j.nantod.2014.09.009

[3] Bhagat, Y., Gangadhara, K., Rabinal, C., Chaudhari, G. and Ugale, P. (2015) Nanotechnology in Agriculture: A Review. Journal of Pure and Applied Microbiology, 9 , 737-747.

[4] Sharon, M., Choudhary, A.K. and Kumar, R. (2010) Nanotechnology in Agricultural Diseases and Food Safety. Journal of Phytology, 2, 83-92.

[5] Prasad, R., Kumar, V. and Prasad, K.S. (2014) Nanotechnology in Sustainable Agriculture: Present Concerns and Future Aspects. African Journal of Biotechnolo$g y$, 13, 705-713. https://doi.org/10.5897/AJBX2013.13554

[6] Rai, M. and Ingle, A. (2012) Role of Nanotechnology in Agriculture with Special Reference to Management of Insect Pests. Applied Microbiology and Biotechnology, 94, 287-293. https://doi.org/10.1007/s00253-012-3969-4

[7] Agrawal, S. and Rathore, P. (2014) Nanotechnology Pros and Cons to Agriculture: A Review. International Journal of Current Microbiology and Applied Sciences, 3 , 43-55.

[8] Servin, A.D. and White, J.C. (2016) Nanotechnology in Agriculture: Next Steps for Understanding Engineered Nanoparticle Exposure and Risk. NanoImpact, 1, 9-12. https://doi.org/10.1016/j.impact.2015.12.002

[9] Schaming, D. and Remita, H. (2015) Nanotechnology: From the Ancient Time to Nowadays. Foundations of Chemistry, 17, 187-205. https://doi.org/10.1007/s10698-015-9235-y

[10] Mukhopadhyay, S.S. (2014) Nanotechnology in Agriculture: Prospects and Constraints. Nanotechnology, Science and Applications, 7, 63. https://doi.org/10.2147/NSA.S39409

[11] Nations, U. (2015) World Population Prospects: The 2015 Revision. United Nations Department of Economic and Social Affairs, 33, 1-66.

[12] Bramhanwade, K., Shende, S., Bonde, S., Gade, A. and Rai, M. (2016) Fungicidal Activity of $\mathrm{Cu}$ Nanoparticles against Fusarium Causing Crop Diseases. Environmental Chemistry Letters, 14, 229-235. https://doi.org/10.1007/s10311-015-0543-1

[13] Chhipa, H. (2017) Nanofertilizers and Nanopesticides for Agriculture. Environmental Chemistry Letters, 15, 15-22. https://doi.org/10.1007/s10311-016-0600-4

[14] Pandey, S., Giri, K., Kumar, R., Mishra, G. and Rishi, R.R. (2018) Nanopesticides: Opportunities in Crop Protection and Associated Environmental Risks. Proceedings of the National Academy of Sciences, India Section B: Biological Sciences, 88, 1287-1308.

[15] Khot, L.R., Sankaran, S., Maja, J.M., Ehsani, R. and Schuster, E.W. (2012) Applications of Nanomaterials in Agricultural Production and Crop Protection: A Review. 
Crop Protection, 35, 64-70. https://doi.org/10.1016/j.cropro.2012.01.007

[16] Ghormade, V., Deshpande, M.V. and Paknikar, K.M. (2011) Perspectives for Nano-Biotechnology Enabled Protection and Nutrition of Plants. Biotechnology Advances, 29, 792-803. https://doi.org/10.1016/j.biotechadv.2011.06.007

[17] Shukla, S.K., Kumar, R., Mishra, R.K., Pandey, A., Pathak, A., Zaidi, M.G.H., Dikshit, A., et al. (2015) Prediction and Validation of Gold Nanoparticles (GNPs) on Plant Growth Promoting Rhizobacteria (PGPR): A Step toward Development of Nano-Biofertilizers. Nanotechnology Reviews, 4, 439-448. https://doi.org/10.1515/ntrev-2015-0036

[18] Liu, R. and Lal, R. (2015) Potentials of Engineered Nanoparticles as Fertilizers for Increasing Agronomic Productions. Science of the Total Environment, 514, 131-139. https://doi.org/10.1016/j.scitotenv.2015.01.104

[19] Ragaei, M. and Sabry, A.K.H. (2014) Nanotechnology for Insect Pest Control. International Journal of Science, Environment and Technology, 3, 528-545.

[20] Mousavi, S.R. and Rezaei, M. (2011) Nanotechnology in Agriculture and Food Production. Journal of Applied Environmental and Biological Sciences, 1, 414-419.

[21] Madhuban, G., Rajesh, K. and Arunava, G. (2012) Nano-Pesticides-A Recent Approach for Pest Control. The Journal of Plant Protection Sciences, 4, 1-7.

[22] Sheykhbaglou, R., Sedghi, M., Shishevan, M.T. and Sharifi, R.S. (2010) Effects of Nano-Iron Oxide Particles on Agronomic Traits of Soybean. Notulae Scientia Biologicae, 2, 112-113. https://doi.org/10.15835/nsb224667

[23] Norman, S. and Hongda, C. (2013) IB in Depth Special Section on Nanobiotechnology, Part 1. Industrial Biotechnology, 9, 17-18.

[24] Nejad, M.S., Bonjar, G.H.S., Khatami, M., Amini, A. and Aghighi, S. (2016) In Vitro and in Vivo Antifungal Properties of Silver Nanoparticles against Rhizoctonia solani, a Common Agent of Rice Sheath Blight Disease. IET Nanobiotechnology, 11, 236-240. https://doi.org/10.1049/iet-nbt.2015.0121

[25] Sidhu, A., Barmota, H. and Bala, A. (2017) Antifungal Evaluation Studies of Copper Sulfide Nano-Aquaformulations and Its Impact on Seed Quality of Rice (Oryzae sativa). Applied Nanoscience, 7, 681-689. https://doi.org/10.1007/s13204-017-0606-7

[26] Ngo, Q.B., Dao, T.H., Nguyen, H.C., Tran, X.T., Van Nguyen, T., Khuu, T.D. and Huynh, T.H. (2014) Effects of Nanocrystalline Powders (Fe, Co and $\mathrm{Cu}$ ) on the Germination, Growth, Crop Yield and Product Quality of Soybean (Vietnamese Species DT-51). Advances in Natural Sciences. Nanoscience and Nanotechnology, 5, Article ID: 015016. https://doi.org/10.1088/2043-6262/5/1/015016

[27] Asghari, F., Jahanshiri, Z., Imani, M., Shams-Ghahfarokhi, M. and Razzaghi-Abyaneh, M. (2016) Antifungal Nanomaterials: Synthesis, Properties, and Applications. In: Grumezescu, A.M., Ed., Nanobiomaterials in Antimicrobial Therapy, Elsevier, Amsterdam, 343-383. https://doi.org/10.1016/B978-0-323-42864-4.00010-5

[28] Bardos, P., Merly, C., Kvapil, P. and Koschitzky, H.P. (2018) Status of Nanoremediation and Its Potential for Future Deployment: Risk-Benefit and Benchmarking Appraisals. Remediation Journal, 28, 43-56. https://doi.org/10.1002/rem.21559

[29] Seok Kim, Y., Ahmad Raston, N.H. and Bock Gu, M. (2016) Aptamer-Based Nanobiosensors. Biosensors and Bioelectronics, 76, 2-19.

https://doi.org/10.1016/j.bios.2015.06.040

[30] Cicek, S. and Nadaroglu, H. (2015) The Use of Nanotechnology in the Agriculture. Advanced Nano Research, 3, 207-223. https://doi.org/10.12989/anr.2015.3.4.207

[31] Rai, V., Acharya, S. and Dey, N. (2012) Implications of Nanobiosensors in Agricul- 
ture. Journal of Biomaterials and Nanobiotechnology, 3, 315.

https://doi.org/10.4236/jbnb.2012.322039

[32] Baruah, S. and Dutta, J. (2009) Nanotechnology Applications in Pollution Sensing and Degradation in Agriculture: A Review. Environmental Chemistry Letters, 7, 191-204. https://doi.org/10.1007/s10311-009-0228-8

[33] Erickson, D., Mandal, S., Yang, A.H.J. and Cordovez, B. (2007) Nanobiosensors: Optofluidic, Electrical and Mechanical Approaches to Biomolecular Detection at the Nanoscale. Microfluidics and Nanofluidics, 4, 33-52. https://doi.org/10.1007/s10404-007-0198-8

[34] Kwak, S.-Y., Wong, M.H., Lew, T.T.S., Bisker, G., Lee, M.A., Kaplan, A., Strano, M.S., et al. (2017) Nanosensor Technology Applied to Living Plant Systems. Annual Review of Analytical Chemistry, 10, 113-140. https://doi.org/10.1146/annurev-anchem-061516-045310

[35] Sarmast, M.K. and Salehi, H. (2016) Silver Nanoparticles: An Influential Element in Plant Nanobiotechnology. Molecular Biotechnology, 58, 441-449. https://doi.org/10.1007/s12033-016-9943-0

[36] Dasgupta, N., Ranjan, S. and Ramalingam, C. (2017) Applications of Nanotechnology in Agriculture and Water Quality Management. Environmental Chemistry Letters, 15, 591-605. https://doi.org/10.1007/s10311-017-0648-9

[37] Dhingra, H.K., Jha, P.N. and Bajpai, M.P. (2011) Current Topics in Biotechnology and Microbiology. Lambert Academic Publishing, Saarbrücken.

[38] Serag, M.F., Kaji, N., Habuchi, S., Bianco, A. and Baba, Y. (2013) Nanobiotechnology Meets Plant Cell Biology: Carbon Nanotubes as Organelle Targeting Nanocarriers. RSC Advances, 3, 4856-4862. https://doi.org/10.1039/c2ra22766e

[39] Vijayalakshmi, C., Chellaram, C. and Kumar, S.L. (2015) Modern Approaches of Nanotechnology in Agriculture-A Review. Biosciences Biotechnology Research Asia, 12, 327-331. https://doi.org/10.13005/bbra/1669

[40] Abd-Elrahman, S.H. and Mostafa, M.A.M. (2015) Applications of Nanotechnology in Agriculture: An Overview. Egyptian Journal of Soil Science, 55, 197-214. https://doi.org/10.21608/ejss.2015.324

[41] Scott, N. and Chen, H. (2013) Nanoscale Science and Engineering for Agriculture and Food Systems. Industrial Biotechnology, 9, 17-18. https://doi.org/10.1089/ind.2013.1555

[42] Bai, H. and Liu, X. (2015) Food Nanotechnology and Nano Food Safety. Nanotechnology Materials and Devices Conference, Anchorage Alaska, 13-16 September 2015, 1-4. https://doi.org/10.1109/NMDC.2015.7439261

[43] Flores-López, M.L., Cerqueira, M.A., de Rodríguez, D.J. and Vicente, A.A. (2016) Perspectives on Utilization of Edible Coatings and Nano-Laminate Coatings for Extension of Postharvest Storage of Fruits and Vegetables. Food Engineering Reviews, 8, 292-305. https://doi.org/10.1007/s12393-015-9135-x

[44] Ramos, O.L., Pereira, R.N., Rodrigues, R., Teixeira, J.A., Vicente, A.A. and Malcata, F.X. (2014) Physical Effects upon Whey Protein Aggregation for Nano-Coating Production. Food Research International, 66, 344-355. https://doi.org/10.1016/j.foodres.2014.09.036

[45] Misra, A.N., Misra, M. and Singh, R. (2013) Nanotechnology in Agriculture and Food Industry. International Journal of Pure and Applied Sciences and Technology, 16,1 .

[46] Chaudhry, Q. and Castle, L. (2011) Food Applications of Nanotechnologies: An 
Overview of Opportunities and Challenges for Developing Countries. Trends in Food Science \& Technology, 22, 595-603. https://doi.org/10.1016/j.tifs.2011.01.001

[47] Magnuson, B.A., Jonaitis, T.S. and Card, J.W. (2011) A Brief Review of the Occurrence, Use, and Safety of Food-Related Nanomaterials. Journal of Food Science, 76, R126-R133. https://doi.org/10.1111/j.1750-3841.2011.02170.x

[48] Sun, S., Sidhu, V., Rong, Y. and Zheng, Y. (2018) Pesticide Pollution in Agricultural Soils and Sustainable Remediation Methods: A Review. Current Pollution Reports, 1-11. https://doi.org/10.1007/s40726-018-0092-x

[49] Gomes, H.I., Fan, G., Mateus, E.P., Dias-Ferreira, C. and Ribeiro, A.B. (2014) Assessment of Combined Electro-Nanoremediation of Molinate Contaminated Soil. Science of the Total Environment, 493, 178-184. https://doi.org/10.1016/j.scitotenv.2014.05.112

[50] Gil-Díaz, M., Diez-Pascual, S., González, A., Alonso, J., Rodríguez-Valdés, E., Gallego, J.R. and Lobo, M.C. (2016) A Nanoremediation Strategy for the Recovery of an As-Polluted Soil. Chemosphere, 149, 137-145. https://doi.org/10.1016/j.chemosphere.2016.01.106

[51] Mueller, N.C. and Nowack, B. (2010) Nanoparticles for Remediation: Solving Big Problems with Little Particles. Elements, 6, 395-400. https://doi.org/10.2113/gselements.6.6.395

[52] Adeleye, A.S., Keller, A.A., Miller, R.J. and Lenihan, H.S. (2013) Persistence of Commercial Nanoscaled Zero-Valent Iron (nZVI) and By-Products. Journal of Nanoparticle Research, 15, 1418. https://doi.org/10.1007/s11051-013-1418-7

[53] Gil-Díaz, M., Gonzalez, A., Alonso, J. and Lobo, M.C. (2016) Evaluation of the Stability of a Nanoremediation Strategy Using Barley Plants. Journal of Environmental Management, 165, 150-158. https://doi.org/10.1016/j.jenvman.2015.09.032

[54] Maine, E., Thomas, V.J., Bliemel, M., Murira, A. and Utterback, J. (2014) The Emergence of the Nanobiotechnology Industry. Nature Nanotechnology, 9, 2. https://doi.org/10.1038/nnano.2013.288

[55] Donaldson, K., Stone, V., Tran, C.L., Kreyling, W. and Borm, P.J. (2004) Nanotoxicology. Occupational and Environmental Medicine, 61, 727-728.

[56] Bennett, D.J. and Schuurbiers, D. (2005) Nanobiotechnology: Responsible Actions on Issues in Society and Ethics. NSTI Nanotech, Vol. 2, 765-768.

[57] Lu, C.M., et al. (2002) Research on the Effect of Nanometer Materials on Germination and Growth Enhancement of Glycine Max and Its Mechanism. Soybean Science, 21, 168-171. 